\title{
Avaliação das pressões venosa e arterial em cães submetidos a diferentes tipos de hipotensão
}

[Evaluation of venous and arterial blood pressures in dogs submitted to hypotension]

\author{
R.C. Rabelo ${ }^{1}$, M.M. Melo ${ }^{1 *}$, P.G. Silva Júnior ${ }^{1}$, M. Lúcia $^{2}$ \\ ${ }^{1}$ Escola de Veterinária - Universidade Federal de Minas Gerais \\ Caixa Postal 567 \\ 30123-970 - Belo Horizonte, MG \\ ${ }^{2}$ Departamento de Biologia - UNIFENAS - Alfenas, MG
}

\begin{abstract}
RESUMO
Estabeleceram-se a pressão venosa periférica (PVP), a pressão venosa central (PVC), a pressão arterial invasiva (PAI) e a pressão arterial não invasiva (PANI) em cães após diferentes eventos de hipotensão. Foram utilizados 15 cães adultos, distribuídos aleatoriamente em três grupos $(\mathrm{G})$ com cinco animais cada, submetidos aos seguintes eventos hipotensores: GI - cloridrato de xilazina a $2 \%$, GII - choque hipovolêmico agudo e GIII - veneno da serpente Bothrops moojeni. Os animais, avaliados durante 30 minutos após o início do evento hipotensor, foram tratados com cloridrato de ioimbina (GI), amido hidroxietílico a 6\% (GII) e cetoprofeno (GIII) e reavaliados por mais 30 minutos. Somente os animais do GII apresentaram redução da PVP após o evento hipotensor e aumento, 25 minutos após tratamento. Os cães dos grupos II e III mostraram redução da PVC após o evento hipotensor, e somente os animais do GII exibiram discreto aumento cinco minutos imediatamente após o tratamento. Houve diminuição da PAI e PANI nos dos grupos II e III após o evento hipotensor, com recuperação gradativa imediata, após o tratamento, somente da PAI.
\end{abstract}

Palavras-chave: cão, xilazina, ioimbina, choque hipovolêmico, amido hidroxietílico, Bothrops moojeni, cetoprofeno

\begin{abstract}
The peripheral venous pressure (PVP), the central venous pressure (CVP), the invasive (IAP) and noninvasive blood pressure (NIAP) in dogs submitted to different hypotensive events were studied. Fifteen adult mongrel dogs were randomly divided in three groups with five animals each, and submitted to hypotensive event as follow: GI - xylazine chloride 2\%, GII - acute hypovolemic shock and GIII - snake venom (Bothrops moojeni). All animals were evaluated for 30 minutes after starting hypotensive event, treated with yoimbine chloride (GI), colloid hetastarch 6\% (GII) and ketoprofen (GIII) and reevaluated for more 30 minutes. Only the group II dogs showed PVP decrease after hypotensive event, and increase 25 minutes after treatment. In animals of groups II and III, the CVP decreased after hypotensive event and only in GII dogs there was mild increase, five minutes after treatment. The groups II and III dogs showed IAP and NIAP decrease after hypotensive event and gradation recuperation immediately after treatment only in IAP.
\end{abstract}

Keywords: dog, xylazine, yoimbine, hypovolemic shock, colloid, Bothrops moojeni, ketoprofen

Recebido para publicação em 9 de julho de 2004

Recebido para publicação, após modificações, em 23 de agosto de 2005

*Autor para correspondência (corresponding author)

E-mail: marilia@vet.ufmg.br 


\section{INTRODUÇÃO}

A casuística de pequenos animais com alterações hipotensivas é freqüente na clínica, superando as alterações hipertensivas. Já que alguns métodos de avaliação da perfusão são considerados de difícil execução e outros de custo elevado, tornase importante avaliar métodos mais acessíveis (Remillard et al., 1991).

Devido à facilidade, baixo custo e possível contribuição na rotina clínica da medicina veterinária, a mensuração da pressão venosa periférica (PVP) e a correlação de seus valores com outras medidas da função hemodinâmica (pressão venosa central, arterial invasiva e não invasiva) podem contribuir na abordagem do paciente crítico.

Trabalhos antigos alertaram para a baixa correlação entre as alterações de volume no choque hemorrágico e a pressão venosa periférica (Wiggers, 1945). Com a publicação de outros estudos (Panke et al., 1959) notou-se que a PVP poderia ter algum significado clínico importante. Naquela época, a avaliação da pressão venosa periférica era a única maneira de tentar prever a condição volêmica do paciente.

Com o advento da cirurgia cardíaca, dos catéteres de linha central e, posteriormente, dos catéteres arteriais, a utilização dos dispositivos intravenosos periféricos ficou restrita à infusão de fluidos e quase não foi relatada na literatura nos últimos 80 anos.

Diante do exposto, este trabalho teve como objetivo avaliar e correlacionar as pressões venosa periférica, venosa central e arterial invasiva e não invasiva em cães após diferentes métodos de hipotensão.

\section{MATERIAL E MÉTODOS}

Utilizaram-se 15 cães adultos jovens, sem raça definida, clinicamente saudáveis, com peso entre 12 e $22 \mathrm{~kg}$, distribuídos aleatoriamente em três grupos contando cinco animais cada um. Esses receberam os seguintes tratamentos hipotensores: grupo I - cloridrato de xilazina ${ }^{1}$ a $2 \%$, na dose de $1,1 \mathrm{mg} / \mathrm{kg}$, via intravenosa lenta e única, capaz de

${ }^{1}$ Dopaser Solução Injetável - Laboratórios Calier S.A. causar estimulação vagal (Greene e Thurmon, 1988); grupo II - retirada de $44 \mathrm{ml}$ de sangue $/ \mathrm{kg}$ (cerca de duas vezes a taxa de coleta de sangue para transfusões sangüíneas) através da veia jugular externa em bolsa de sangue apropriada ${ }^{2}$, aproximadamente em dois minutos, no intuito de ocasionar choque hipovolêmico agudo (Aird, 2000); grupo III - administração de veneno da serpente Bothrops moojeni, na dose de $0,3 \mathrm{mg} / \mathrm{kg}$, via intravenosa lenta, diluído em solução salina $(0,3 \mathrm{~g}$ de veneno para $100 \mathrm{ml}$ de cloreto se sódio a 0,9\%), em dose única (Sano-Martins et al., 1995). Trinta minutos após as mensurações e avaliações propostas, os animais foram tratados com o cloridrato de ioimbina ${ }^{3}$ na dose de $1,1 \mathrm{mg} / \mathrm{kg}$, via intravenosa lenta e única (GI), amido hidroxietílico ${ }^{4}$ (composto colóide expansor plasmático), na dose de $20 \mathrm{ml} / \mathrm{kg} / \mathrm{hora}$ via intravenosa, infundido em 30 minutos por meio de bomba de infusão ${ }^{5}$, que resultou na dose total de $10 \mathrm{ml} / \mathrm{kg}$ (GII) e com cetoprofeno ${ }^{6}$, antiinflamatório não esteróide, na dose de $2 \mathrm{mg} / \mathrm{kg}$ na concentração de $1 \%$, via intravenosa lenta e única (GIII). Após os tratamentos, os animais foram avaliados por mais 30 minutos.

O intervalo de tempo $(\mathrm{T})$ entre as avaliações foi de cinco minutos durante 60 minutos. A primeira avaliação, T0, ocorreu 24 horas após a colocação dos catéteres; a segunda (T1), imediatamente após a infusão do protocolo de sedação proposto; a terceira (T2), imediatamente após o início do evento hipotensor; da quarta à sexta avaliações (T5, T10, T15, T20, T25 e T30) ocorreram cinco minutos após T2. Em T30, após a avaliação dos animais, iniciou-se o tratamento proposto para o evento hipotensor. A partir daí, e a cada cinco minutos (T35, T40, T45, T50, T55 e T60), os animais foram reavaliados.

Vinte e quatro horas antes de se iniciar o experimento, todos os animais foram canulados na artéria carótida esquerda, na veia jugular esquerda e na veia cefálica esquerda. A sedação foi realizada com acepromazina ${ }^{7}$ pela via intramuscular $(0,25 \mathrm{mg} / \mathrm{kg})$. Após 30 minutos,

\footnotetext{
${ }^{2}$ Modelo CPDA - JP Indústria Farmacêutica

${ }^{3}$ Cloridrato de ioimbina - Manipulação, Laboratórios Duprat Ltda - RJ

${ }^{4}$ HES-Steril ${ }^{\circledR}-6 \%$ de Hetastarch em solução salina

fisiológica - Fresenius Kabi

${ }^{5}$ Samtronic $550 \mathrm{~T} 2$

${ }^{6}$ Ketofen 1\% - Merial Saúde Animal Ltda, SP

${ }^{7}$ Acepran 1\% - UNIVET S.A. Ind. Vet. - SP
} 
fez-se o preparo cirúrgico da face cranial do membro anterior esquerdo, face cervical ventral e lateral esquerda, com tricotomia e anti-sepsia com álcool e iodopovidina.

Para fins de infusão do tratamento e das drogas sedativas, canulou-se a veia cefálica direita (cateter intravenoso periférico ${ }^{8}$ ) após preparo cirúrgico da área. Posteriormente, realizou-se sedação intravenosa com fentanil ${ }^{9}(0,05 \mathrm{mg} / \mathrm{kg})$ e diazepan ${ }^{10}(0,5 \mathrm{mg} / \mathrm{kg})$ correspondente ao $\mathrm{T} 1 \mathrm{e}$ anestesia local por botão com 1,0ml de lidocaína $2 \%{ }^{11}$, sem vasoconstritor.

Para mensuração da pressão venosa central (PVC), após preparo cirúrgico da área, a veia jugular foi isolada por dissecação e ligada em sua porção anterior com fio de náilon 2-0. Após incisão com lâmina de bisturi número 11, posicionou-se uma sonda nasogástrica longa $12 \mathrm{G}$ de poliuretano ${ }^{12}$, para introdução de cânula na veia jugular e introduzida até a veia cava cranial. A mensuração deu-se com o uso de dois equipos macrogotas e uma torneira de três vias. A leitura foi feita por meio de uma coluna de água, e a medida dada em centímetros (Soares et al., 2000).

Para mensuração da pressão venosa periférica (PVP), puncionou-se a veia cefálica esquerda com cateter periférico de longa permanência $20 \mathrm{G}^{13}$. Um cateter foi conectado a um sistema de dois equipos e uma torneira de três vias e a PVP mensurada por meio de coluna d'água, em centímetros de água, semelhante à mensuração realizada no acesso central.

A pressão arterial não invasiva (PANI) foi avaliada por oscilometria. Utilizou-se um monitor de pressão arterial não invasivo ${ }^{14}$, multiparamétrico. Um manguito com largura de aproximadamente $40 \%$ da circunferência do membro foi colocado ao redor da porção caudal, próxima da articulação úmero-rádio-ulnar, do radioulna, do membro anterior direito. $\mathrm{O}$ aparelho realizou as medições automaticamente.

\footnotetext{
${ }^{8}$ Insyte $20 \mathrm{G} \mathrm{BD}^{\circledR}$

${ }^{9}$ Fentanil - Cristália Laboratórios

${ }^{10}$ Diazepan

${ }^{11}$ Lidocaína 2\% - Lidovet - Laboratório Bravet Ltda - RJ

${ }^{12}$ Sonda Nasogástrica Longa Sanobiol

${ }^{13}$ Safe-T Intima $\mathrm{BD}^{\circledR}$ - Beckton Dikinson

${ }^{14}$ Emai RX-300
}

Para mensuração da pressão arterial invasiva (PAI), localizou-se e dissecou-se a artéria carótida esquerda e, após o isolamento, ela foi ligada em sua porção cranial, com fio de náilon 2-0, e incisada com lâmina de bisturi número 11 , para inserção da prótese (dispositivo intravenoso central $12 \mathrm{G})^{15}$.

O delineamento experimental preconizado foi o de parcelas subdivididas, de acordo com Sampaio (1998). Os dados referentes aos diferentes grupos e tempos foram plotados em tabelas e analisados pelo programa de computador SAS (User's... 1985). As diferenças mínimas significativas para todas as variáveis estudadas foram comparadas pelo teste $t$ de Student entre grupos e tempos, com nível de significância de $\mathrm{P} \leq 0,05$.

\section{RESULTADOS E DISCUSSÃO}

Os resultados são mostrados nas Tab. 1, 2, 3 e 4 e na Fig. 1.

Tabela 1. Valores médios da pressão venosa periférica (PVP) $\left(\mathrm{cmH}_{2} \mathrm{O}\right)$ de cães em diferentes tempos $(\mathrm{T})$ e condições hemodinâmicas (grupo)

\begin{tabular}{lccc}
\hline \multicolumn{3}{c}{ PVP $\left(\mathrm{cm} \mathrm{H}_{2} \mathrm{O}\right)$} \\
\hline Tempo & Grupo I & Grupo II & Grupo III \\
\hline T0 & $7,7 \mathrm{Acd}$ & $9,4 \mathrm{Abc}$ & $9,3 \mathrm{Abc}$ \\
T1 & $10,8 \mathrm{Ac}$ & $9,5 \mathrm{Abc}$ & $12,7 \mathrm{Aa}$ \\
T2 & $14,2 \mathrm{Aab}$ & $9,1 \mathrm{Abc}$ & $11,4 \mathrm{Aab}$ \\
T5 & $14,5 \mathrm{Aa}$ & $8,5 \mathrm{Bc}$ & $10,3 \mathrm{Aabc}$ \\
T10 & $12,9 \mathrm{Aabc}$ & $9,5 \mathrm{Abc}$ & $10,2 \mathrm{Aabc}$ \\
T15 & $12,2 \mathrm{Aabc}$ & $9,2 \mathrm{Abc}$ & $9,9 \mathrm{Abc}$ \\
T20 & $10,7 \mathrm{Ac}$ & $10,8 \mathrm{Aabc}$ & $9,7 \mathrm{Abc}$ \\
T25 & $11,0 \mathrm{Ac}$ & $11,0 \mathrm{Aabc}$ & $10,5 \mathrm{Aabc}$ \\
T30 & $10,8 \mathrm{Ac}$ & $11,0 \mathrm{Aabc}$ & $9,4 \mathrm{Abc}$ \\
T35 & $11,0 \mathrm{Ac}$ & $11,6 \mathrm{Aab}$ & $9,4 \mathrm{Abc}$ \\
T40 & $10,8 \mathrm{Ac}$ & $11,5 \mathrm{Aab}$ & $9,5 \mathrm{Abc}$ \\
T45 & $11,1 \mathrm{Ac}$ & $11,3 \mathrm{Aab}$ & $9,2 \mathrm{Abc}$ \\
T50 & $11,8 \mathrm{Abc}$ & $13,3 \mathrm{Aa}$ & $8,7 \mathrm{Ac}$ \\
T55 & $11,6 \mathrm{Abc}$ & $13,4 \mathrm{Aa}$ & $9,0 \mathrm{Abc}$ \\
T60 & $11,4 \mathrm{Ac}$ & $12,9 \mathrm{Aa}$ & $9,1 \mathrm{Abc}$ \\
\hline
\end{tabular}

Médias seguidas de letras maiúsculas distintas na linha e minúsculas distintas na coluna diferem entre si $(P \leq 0,05)$. CV: $22,7 \%$.

Grupo I - cloridrato de xilazina a $2 \%$ e tratamento com cloridrato de ioimbina; grupo II - choque hipovolêmico agudo e tratamento com amido hidroxietílico a 6\%; e grupo III - veneno da serpente Bothrops moojeni e tratamento com cetoprofeno.

\footnotetext{
${ }^{15}$ Cateter intravenoso Intracath $\mathrm{BD}^{\circledR}$ - Beckton Dikinson
} 
Tabela 2. Valores médios da pressão venosa central (PVC) $\left(\mathrm{cmH}_{2} \mathrm{O}\right)$ de cães em diferentes tempos $(\mathrm{T})$ e condições hemodinâmicas (grupo)

\begin{tabular}{lccc}
\hline \multicolumn{3}{c}{ PVC $\left(\mathrm{cmH}_{2} \mathrm{O}\right)$} \\
\hline Tempo & Grupo I & Grupo II & Grupo III \\
\hline T0 & $4,4 \mathrm{Ae}$ & $1,9 \mathrm{Aab}$ & $2,2 \mathrm{Aab}$ \\
T1 & $7,1 \mathrm{Abcd}$ & $4,5 \mathrm{ABa}$ & $2,9 \mathrm{Bab}$ \\
T2 & $7,4 \mathrm{Abc}$ & $-1,6 \mathrm{Bd}$ & $0,9 \mathrm{Bb}$ \\
T5 & $10,6 \mathrm{Aa}$ & $-1,2 \mathrm{Bd}$ & $2,7 \mathrm{Cab}$ \\
T10 & $8,6 \mathrm{Aab}$ & $-1,2 \mathrm{Bd}$ & $3,7 \mathrm{Ca}$ \\
T15 & $8,0 \mathrm{Aab}$ & $-1,0 \mathrm{Bcd}$ & $3,3 \mathrm{Cab}$ \\
T20 & $7,1 \mathrm{Abcd}$ & $-1,0 \mathrm{Bcd}$ & $2,6 \mathrm{Cab}$ \\
T25 & $7,3 \mathrm{Abc}$ & $-0,8 \mathrm{Bcd}$ & $2,5 \mathrm{Bab}$ \\
T30 & $6,2 \mathrm{Abcde}$ & $-0,4 \mathrm{Bcd}$ & $3,5 \mathrm{Aab}$ \\
T35 & $5,3 \mathrm{Acde}$ & $0,4 \mathrm{ABbcd}$ & $3,5 \mathrm{Aab}$ \\
T40 & $4,6 \mathrm{Ade}$ & $-0,2 \mathrm{Bbcd}$ & $3,9 \mathrm{Aa}$ \\
T45 & $5,0 \mathrm{Acde}$ & $-0,2 \mathrm{Bbcd}$ & $3,5 \mathrm{Aab}$ \\
T50 & $5,0 \mathrm{Acde}$ & $-0,2 \mathrm{Bbcd}$ & $3,7 \mathrm{Aa}$ \\
T55 & $4,6 \mathrm{Ade}$ & $-0,2 \mathrm{ABbcd}$ & $2,8 \mathrm{Aab}$ \\
T60 & $5,0 \mathrm{Acde}$ & $1,6 \mathrm{Abc}$ & $4,1 \mathrm{Aa}$ \\
\hline
\end{tabular}

Médias seguidas de letras maiúsculas distintas na linha minúsculas distintas na coluna diferem entre si $(\mathrm{P} \leq 0,05)$. CV: $68,0 \%$

Grupo I - cloridrato de xilazina a $2 \%$ e tratamento com cloridrato de ioimbina; grupo II - choque hipovolêmico agudo e tratamento com amido hidroxietílico a $6 \%$; e grupo III - veneno da serpente Bothrops moojeni e tratamento com cetoprofeno.

Tabela 3. Valores médios da pressão arterial invasiva (PAI) $(\mathrm{mmHg})$ de cães em diferentes tempos (T) e condições hemodinâmicas (grupos)

\begin{tabular}{lccc}
\hline \multicolumn{3}{c}{ PAI $(\mathrm{mmHg})$} \\
\hline Tempo & Grupo I & Grupo II & Grupo III \\
\hline T0 & $108 \mathrm{Abcd}$ & $107 \mathrm{Aa}$ & $119 \mathrm{Aa}$ \\
T1 & $111 \mathrm{Aabcd}$ & $105 \mathrm{Ba}$ & $109 \mathrm{Aba}$ \\
T2 & $121 \mathrm{Aab}$ & $59 \mathrm{Bf}$ & $72 \mathrm{Bcd}$ \\
T5 & $125 \mathrm{Aa}$ & $59 \mathrm{Bf}$ & $66 \mathrm{Bd}$ \\
T10 & $123 \mathrm{Aab}$ & $61 \mathrm{Cf}$ & $92 \mathrm{Bbc}$ \\
T15 & $114 \mathrm{Aabc}$ & $69 \mathrm{Bdef}$ & $92 \mathrm{ABbc}$ \\
T20 & $113 \mathrm{Aabcd}$ & $71 \mathrm{Bdef}$ & $107 \mathrm{Aab}$ \\
T25 & $114 \mathrm{Aabc}$ & $69 \mathrm{Bdef}$ & $108 \mathrm{Aa}$ \\
T30 & $121 \mathrm{Aab}$ & $66 \mathrm{Bef}$ & $110 \mathrm{Aa}$ \\
T35 & $105 \mathrm{Acd}$ & $75 \mathrm{Bcde}$ & $114 \mathrm{Aa}$ \\
T40 & $98 \mathrm{Abd}$ & $73 \mathrm{Acdef}$ & $114 \mathrm{Ba}$ \\
T45 & $98 \mathrm{Abd}$ & $81 \mathrm{Abcde}$ & $108 \mathrm{Ba}$ \\
T50 & $102 \mathrm{Abcd}$ & $82 \mathrm{Abcd}$ & $111 \mathrm{Ba}$ \\
T55 & $99 \mathrm{Acd}$ & $87 \mathrm{Acd}$ & $113 \mathrm{Aa}$ \\
T60 & $101 \mathrm{Acd}$ & $95 \mathrm{Aab}$ & $114 \mathrm{Aa}$ \\
\hline Médias & seguidas de letras & maiúsculas distintas na linha
\end{tabular}

Médias seguidas de letras maiusculas distintas na linha e
minúsculas distintas na coluna diferem entre si $(\mathrm{P} \leq 0,05)$. CV: $13,4 \%$.

Grupo I - cloridrato de xilazina a $2 \%$ e tratamento com cloridrato de ioimbina; grupo II - choque hipovolêmico agudo e tratamento com amido hidroxietílico a $6 \%$; e grupo III - veneno da serpente Bothrops moojeni e tratamento com cetoprofeno.
Tabela 4. Valores médios da pressão arterial não invasiva (PANI) (mmHg) de cães em diferentes tempos (T) e condições hemodinâmicas (grupos)

\begin{tabular}{lccc}
\hline \multicolumn{4}{c}{ PANI $(\mathrm{mmHg})$} \\
\hline Tempo & Grupo I & Grupo II & Grupo III \\
\hline T0 & $108 \mathrm{Aa}$ & $97 \mathrm{Aab}$ & $120 \mathrm{Aa}$ \\
T1 & $109 \mathrm{Aa}$ & $115 \mathrm{Aa}$ & $107 \mathrm{Aab}$ \\
T2 & $109 \mathrm{Aa}$ & $76 \mathrm{Bbcdef}$ & $71 \mathrm{Bef}$ \\
$\mathrm{T} 5$ & $121 \mathrm{Aa}$ & $57 \mathrm{Bef}$ & $66 \mathrm{Bf}$ \\
$\mathrm{T} 10$ & $118 \mathrm{Aa}$ & $52 \mathrm{Bf}$ & $73 \mathrm{Bdef}$ \\
T15 & $114 \mathrm{Aa}$ & $72 \mathrm{Bbcdef}$ & $83 \mathrm{Bcdef}$ \\
T20 & $111 \mathrm{Aa}$ & $61 \mathrm{Bdef}$ & $86 \mathrm{ABbcdef}$ \\
$\mathrm{T} 25$ & $109 \mathrm{Aa}$ & $90 \mathrm{Aabc}$ & $94 \mathrm{Aabcde}$ \\
T30 & $111 \mathrm{Aa}$ & $74 \mathrm{Bbcdef}$ & $103 \mathrm{ABabc}$ \\
T35 & $103 \mathrm{Aa}$ & $86 \mathrm{Abcd}$ & $99 \mathrm{Aabcd}$ \\
T40 & $97 \mathrm{Aa}$ & $69 \mathrm{Acdef}$ & $99 \mathrm{Aabcd}$ \\
T45 & $99 \mathrm{Aa}$ & $71 \mathrm{Abcdef}$ & $99 \mathrm{Aabcd}$ \\
T50 & $99 \mathrm{Aa}$ & $74 \mathrm{Abcdef}$ & $99 \mathrm{Aabcd}$ \\
T55 & $99 \mathrm{Aa}$ & $80 \mathrm{Abcde}$ & $101 \mathrm{Aabc}$ \\
T60 & $97 \mathrm{Aa}$ & $82 \mathrm{Abcde}$ & $98 \mathrm{Aabcd}$ \\
\hline
\end{tabular}

Médias seguidas de letras maiúsculas distintas na linha e minúsculas distintas na coluna diferem entre si $(\mathrm{P} \leq 0,05)$. CV: $23,3 \%$.

Grupo I - cloridrato de xilazina a $2 \%$ e tratamento com cloridrato de ioimbina; grupo II - choque hipovolêmico agudo e tratamento com amido hidroxietílico a $6 \%$; e grupo III - veneno da serpente Bothrops moojeni e tratamento com cetoprofeno.

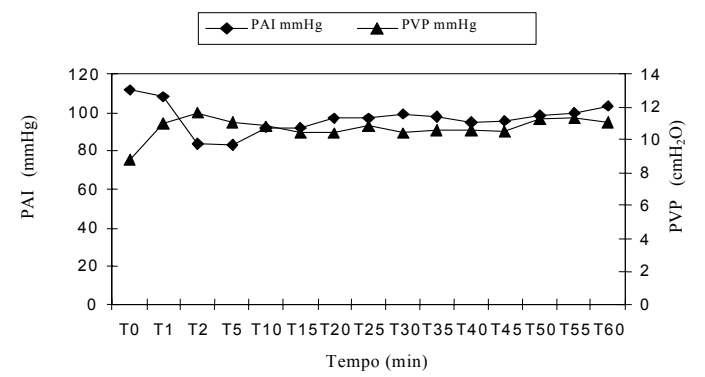

Figura 1. Correlação entre pressão arterial invasiva (PAI) $(\mathrm{mmHg})$ e pressão venosa periférica (PVP) $\left(\mathrm{cmH}_{2} \mathrm{O}\right)$ sob diferentes condições hemodinâmicas, em diferentes tempos.

No GI, houve aumento da PVP $(\mathrm{P} \leq 0,05)$ imediatamente após o evento hipotensor (Tab. 1). Em T10, houve redução da PVP que se manteve sem alteração estatística até T60. No GII, após evento hipotensor, a PVP não alterou. Possivelmente, a perda imediata de volume sangüíneo foi antagonizada pelo mecanismo compensatório do leito venoso e, após esses primeiros minutos, o leito venoso e seu mecanismo compensatório fizeram-se úteis ao organismo quando houve constrição e tentativa 
de envio de líquido para a linha central observada nos tempos T10, T15, T20, T25 e T30. Esse mecanismo teve a ação sinérgica do colóide administrado em T30, com recuperação de líquido intersticial e aumento de pressão coloidosmótica em associação com o tônus venoso e a PVP. Em T60, a PVP ainda apresentou valores significativamente mais altos quando comparados com T0, T1, T2, T5, T10 e T15, possivelmente devido à estimulação simpática decorrente da redução de pressão e ao choque, na tentativa de manter a perfusão dos órgãos nobres por meio da impulsão de sangue obtida pela vasoconstrição (Tab. 1). Deve-se ressaltar que, nesse momento, uma rápida infusão de líquido pelo sistema venoso periférico poderia ser altamente prejudicial ao organismo que se encontra sob alta pressão. Uma terapêutica adequada no momento do choque seria a verificação do volume central por meio da PVC e a infusão de líquidos nessa mesma linha. No GIII, não houve diminuição significativa da PVP após administração do veneno botrópico (Tab. 1). Como não houve perda de líquido ou sangue neste grupo em todos os tempos avaliados, a força contrátil do sistema venoso foi capaz de controlar e manter a PVP.

A pressão venosa central apresentou tendência diferente entre os grupos estudados (Tab. 2). No GI houve aumento da PVC logo após a infusão do cloridrato de xilazina, que culminou em hipertensão venosa central significativa $(\mathrm{P} \leq 0,05)$ em T5. Esse fenômeno, já relatado por Cortopani e Fantoni (2002), ocorreu provavelmente devido à hipertensão pulmonar gerada pela xilazina nos minutos iniciais após a sua administração. Após esse período, a PVC apresentou uma redução progressiva que se manteve durante o tratamento com cloridrato de ioimbina e atingiu os valores observados no T0 ao final da avaliação (T60). No GII, ocorreu redução brusca e imediata $(\mathrm{P} \leq 0,05)$ da PVC logo após a retirada do sangue (Tab. 2), também esperada devido à relação direta da PVC com o volume circulante (Oakley et al., 1997). Até o início da terapia com o colóide (T30), o organismo não foi capaz de alterar os valores de PVC por mecanismo de compensação (Raiser, 2005). A partir da infusão do colóide (T35), houve leve incremento da PVC com discreta redução subseqüente (T40), que se manteve até $\mathrm{T} 55$, muito provavelmente porque o volume infundido não foi suficiente para preencher o leito venoso (Crowe e Devey, 1994;
Rudloff e Kirby, 1998; Nolan, 2001). Porém em $\mathrm{T} 60$, apesar de não significativa, reiniciou-se a elevação da PVC, nova tentativa do organismo em restabelecer a PVC, em resposta ao tratamento estabelecido (colóide), com conseqüente aumento da pressão coloidosmótica e do recrutamento de líquido intersticial para o meio intravascular. Ressalta-se que o HESSteril ${ }^{\circ}$ é o colóide de maior meia-vida, mas de menor velocidade de expansão imediata, justificando a resposta encontrada (Chan et al., 2001; Cortopassi, 2002). No GIII, houve redução rápida da PVC, imediatamente após a administração do veneno botrópico (T2), atribuída à ação vasomotora imediata provocada pelos mediadores da hipotensão nele presentes (Roodt et al., 1996). Logo após esse evento, houve recuperação progressiva e significativa da PVC $(\mathrm{P} \leq 0,05)$ em T5, mantida após o tratamento com cetoprofeno, diferente de Silva Júnior (2003), ao relatar que a redução da PVC no envenenamento botrópico, sem qualquer tipo de tratamento, é rápida e não é suprimida até, pelo menos, $3 \mathrm{~h}$ após o evento hipotensor. Esse efeito de recuperação sugere também que não houve perda significativa de volume intravascular durante os 60 minutos de observação do fenômeno ou, ainda, que os mecanismos de contração vascular compensatórios foram suficientes para manter a condição volêmica intravascular, haja vista que a pressão venosa periférica não apresentou alterações significativas no mesmo período.

Observou-se baixa correlação entre PVP e PVC, quando se compararam os valores médios absolutos: correlações negativas de 7,4\% (GII) e $16,0 \%$ (GIII) e correlação positiva de $26,9 \%$ no GI.

Nos animais do GI, observou-se aumento da PVP em conjunto com a PVC, explicado pela redução na freqüência cardíaca e no débito cardíaco além de hipertensão pulmonar, causadores de aumento da congestão venosa central e periférica.

Nos grupos II e III, não se observou correlação entre as pressões, porém verificou-se que, quando há redução na pressão venosa central devido à hemorragia ou mesmo por dilatação, há uma compensação imediata do sistema venoso periférico por meio de vasoconstrição, com conseqüente aumento na PVP. Essa alteração ocorre principalmente nos momentos iniciais do 
evento hipotensor e fornece importante informação sobre o estado geral do paciente.

A pressão arterial invasiva, que fornece o valor da pressão arterial média (PAM), apresentou tendência diferente entre os grupos estudados (Tab. 3). Em vários momentos, durante o estudo, houve diferença significativa $(\mathrm{P} \leq 0,05)$ nas comparações grupo a grupo, mostrando os diferentes comportamentos do organismo frente a eventos hipotensores de origens diferentes.

No GI, houve leve hipertensão arterial média, $(\mathrm{P} \leq 0,05)$ em $\mathrm{T} 5$, somente quando comparado com os valores médios de T0 (Tab. 3). Após o tratamento com o cloridrato de ioimbina em T35, houve redução $(\mathrm{P} \leq 0,05)$, que se manteve até T60. De maneira geral, essa variável comportou-se como sugere a literatura quanto ao aumento inicial logo após a infusão da xilazina (Cortopani e Fantoni, 2002), que durou cerca de 15 minutos, mas não houve a hipotensão esperada após esse período. Esse fato pode ser atribuído à maior estimulação simpática imposta aos animais desse grupo, ao estresse aumentado ou a outros fatores relacionados à sensibilidade exercida pela xilazina, fatores que interferiram na ação hipotensora do cloridrato de xilazina.

Nos animais do GII, houve hipotensão imediatamente após a retirada do sangue total (T2), resultando em choque acompanhado de seus sinais clínicos mais comuns (pulso fraco, mucosas pálidas e secas, extremidades frias, respiração agônica e até mesmo alterações de consciência) (Crowe e Devey, 1994; Rudloff e Kirby, 1994; Nolan, 2001). Após a infusão do fluido colóide (T30), houve recuperação gradativa da pressão (Tab. 4), demostrando diferença após cinco minutos de infusão e retornando aos valores normais de pressão arterial média em T60, sem causar qualquer tipo de sinal de edema ou efeito colateral (Marik e Iglesias, 2000; Ablij et al., 2001; Chan et al., 2001; Cortopassi, 2002).

Mesmo na dose mínima recomendada de $10 \mathrm{ml} / \mathrm{kg} / \mathrm{h}$, o colóide mostrou-se efetivo no restabelecimento da normalidade da pressão média. É importante ressaltar que, apesar de a literatura oferecer limites de 10 a $20 \mathrm{ml} / \mathrm{kg} / \mathrm{h}$ (Mazaferro et al., 2002), clinicamente, a dose de ataque utilizada é sempre de $20 \mathrm{ml} / \mathrm{kg} / \mathrm{h}$, nos casos de choque hemorrágico. Durante a recuperação dos valores fisiológicos de pressão arterial média, todos os sinais associados ao choque (pulso fraco, mucosas pálidas e secas, extremidades frias, respiração agônica e alterações de consciência) foram suprimidos.

Os animais do GIII apresentaram hipotensão com choque subseqüente, sinais esperados, tanto pela ação hipotensora como pela lesão vascular, provocados pelo veneno (Roodt et al., 1996). Ressaltam-se os mecanismos compensatórios do organismo, pois a partir de T10 observou-se discreta recuperação da pressão arterial média. A partir de T20 e até T60, os valores da pressão arterial foram semelhantes aos de T0. Como a pressão arterial neste grupo retornou aos valores basais antes do tratamento, possivelmente $\mathrm{o}$ cetoprofeno (aplicado após T30) auxiliou somente na manutenção dos valores normais da pressão arterial. Sano-Martins et al. (1995) avaliaram a PAI em cães após envenenamento botrópico e verificaram redução progressiva e grave dessa. Como os resultados desta pesquisa diferem dos de Sano-Martins et al. (1995), sugere-se que o cetoprofeno tenha uma possível ação reversora sobre a hipotensão no envenenamento botrópico. Nesse grupo, a manifestação clínica dos sinais de choque não foi tão evidente, mas a presença de pulso fraco e de extremidades frias ocorreu em alguns animais, sinais esses suprimidos juntamente com a redução da pressão arterial, por compensação do organismo, após T10.

A correlação estimada entre PVP e PAI indicou variação das tendências, de acordo com o tipo de evento hipotensor. No caso da infusão de cloridrato xilazina nos animais do GI, observouse correlação positiva, 38,6\%; nos animais do GII (choque hemorrágico), a correlação foi negativa, $38,3 \%$, e no envenenamento botrópico (GIII), o valor foi positivo, 18,1\%. É possível notar que as correlações nos grupos I e II acompanharam o padrão de relação das variáveis quando se comparou PVC com PVP. Na infusão de cloridrato de xilazina, ocorre hipertensão inicial simultânea à redução na freqüência cardíaca e no débito cardíaco, além da hipertensão pulmonar, gerando aumento das duas variáveis. No GII, verificou-se correlação negativa, esperada devido à compensação do organismo, por vasoconstrição periférica, no sentido de desviar volume sangüíneo para a circulação central e combater a hipotensão 
arterial e venosa central. No GIII, observou-se correlação menor e positiva e, provavelmente, isso ocorreu pela maior facilidade do organismo em compensar a hipotensão observada nesse caso, devido à melhor acomodação venosa do evento hipotensor por envenenamento botrópico do que no choque hemorrágico. Esse tipo de compensação faz com que a avaliação da PVP deixe dúvidas, por não variar em sua total extensão.

A obtenção da PANI, tomada pelo monitor multiparamétrico, depende da qualidade do pulso e do ritmo cardíaco para se realizar medidas adequadas. Por isso, pouco se utilizam os valores da PANI junto com os da PAI, nos casos de choque, pois há interferência, na maioria dos monitores oscilométricos, sobre a qualidade das mensurações (Jones, 1996a), e os valores oscilam em torno de $10 \mathrm{mmHg}$ (mais baixos que em medidas diretas) (Grosenbaugh e Muir, 1998). Além disso, alguns monitores não indicam limite de referência para pequenos animais, o que pode prejudicar sua utilização (Grosenbaugh e Muir, 1998).

Neste estudo, de modo geral, a mensuração da PANI mostrou tendência semelhante à realizada pela PAI, quanto aos eventos fisiológicos ocorridos. Nos animais do GI, durante todo o tempo estudado, não houve alteração significativa (Tab. 4). Os animais dos grupos II e III apresentaram redução da PANI, imediatamente após evento hipotensor (T2). Nos do GII, somente 30 minutos (T60) após o tratamento com colóide (T30) observou-se diferença $(\mathrm{P} \leq 0,05)$. Os cães do GIII mostraram aumento da PANI $(\mathrm{P} \leq 0,05)$ em $\mathrm{T} 10$, antes do tratamento com cetoprofeno.

A correlação entre PAI e PANI nos grupos II e III não foi alta ( $48 \%$ e $47 \%$, respectivamente), mas, ao contrário, no grupo I, foi elevada, $90 \%$. Neste estudo, a mensuração da PANI não seguiu linearmente as características sugeridas por Vincent et al. (1993) e Grosenbaugh e Muir (1998), mostrando valores mais baixos, iguais ou acima da PAI. Dependendo do momento da mensuração, a correlação geral entre os métodos em todas as situações provocadas foi $66 \%$, valor que suporta a utilização da PANI na rotina clínica, como método de mensuração da pressão arterial. Os dados reforçam a tese de que essa medida não invasiva depende significativamente da qualidade de pulso arterial (Jones, 1996).

Quando se correlacionam as variáveis, em todos os eventos hipotensores, observa-se tendência clínica esperada, ou seja, a PVP apresenta-se mais alta nos momentos de hipotensão arterial e venosa, devido à tentativa de impulsionar volume até os leitos sangüíneos importantes.

Segundo Yeomans et al. (1943) e Warren et al. (1945), a PVP só apresenta mudanças drásticas em situações de colapso circulatório agudo. A afirmação de Landis e Hortenstine (1950) de que a PVP era imprecisa, provavelmente, foi recebida pela comunidade científica da época como uma verdade sem contestação. Por essa razão, a PVP ficou esquecida no tempo por 60 anos, desde os últimos relatos em 1944. Interpretando seus valores com senso crítico e clínico, é possível extrair informações importantes sobre a condição geral do paciente, associando-os aos parâmetros clínicos (freqüência cardíaca, pulso e coloração das mucosas).

Sabe-se que a pressão periférica é controlada pelo volume intravascular, pela contratilidade venosa e pelas pressões dos grandes compartimentos (pressão intratorácica e intrabdominal) (Rabelo, 2003). É preciso identificar os fatores envolvidos no choque, a história prévia do paciente e, juntamente com a PVP, estimar a condição hemodinâmica do paciente até que outros métodos de avaliação, mais precisos, possam ser utilizados.

A medida da pressão venosa periférica é um método simples, que pode ser feito pela punção de qualquer vaso periférico, capaz de antecipar, para o clínico, o momento no qual o organismo inicia a descompensação hemodinâmica, principalmente nas situações em que o paciente não esteja sob monitoração intensiva e naquelas que não envolvam o estado de choque, com vasoconstrição, além de mostrar-se fidedigna às variações da pressão venosa central e da pressão arterial invasiva.

Conclui-se que, após o evento hipotensor grave, como choque hipovolêmico, há rápida redução da PVP, PVC, PAI e PANI e, 30 minutos após o tratamento específico, só é possível detectar aumento da PAI. 


\section{AGRADECIMENTOS}

Os autores agradecem ao Dr. Dennis T. Crowe e à Dra. Jennifer Devey pelo auxílio nesta pesquisa.

\section{REFERÊNCIAS BIBLIOGRÁFICAS}

ABLIJ, H.C.; INNEMME, G.; TAMSMA, J.T. et al. Intravenous fluid therapy taken into theoretical and practical consideration: physiology revisited. Neth. J. Med., v.58, p.111-122, 2001 .

AIRD, B. Acute blood loss anemia. In: Schalm's veterinary hematology. 5.ed. Philadelphia: Lippincott Willians \& Wilkins, 2000. 1344p.

CHAN, D.L.; FREEMAN, L.M.; ROZANSKY, E.A. Colloid osmotic pressure of parenteral nutrition components and intravenous fluids. J. Vet. Emerg. Crit. Care, v.11, p.269273, 2001.

CORTOPASSI, S.R.G. Fluidoterapia na anestesia. In: FANTONI, D.T.; CORTOPASSI, S.R.G. Anestesia em cães e gatos. Roca: São Paulo, 2002. 430p.

CROWE, D.T.; DEVEY, J.J. Assessment and management of the hemorrhaging patient. Vet. Clin. North Am.: Small Anim. Pract., v.24, p.1095-1121, 1994.

GREENE, S.A.; THURMON, J.C. Xylazine - a review of its pharmacology and use in veterinary medicine. J. Vet. Pharmacol. Therap., v.11, p.295-313, 1988.

GROSENBAUGH, D.A.; MUIR, W.W. Blood pressure monitoring. Vet. Med., v.38, p.48-59, 1998.

JONES, J.L. Non-invasive monitoring techniques in anesthesized animals. Vet. Med., v.91, p.326-336, 1996.

LANDIS, E.M.; HORTENSTINE, J.C. Functional significance of venous blood pressure. Physiol. Rev., v.30, p.1-32, 1950.

MARIK, P.E.; IGLESIAS, J. Would the colloid detractors please sit down! Crit. Care Med., v.28, p.2654-2654, 2000.

MAZZAFERRO, E.M.; RUDLOFF, E.R.; KIRBY, R. The role of albumin replacement in the critically ill veterinary patient. J. Vet. Emerg. Crit. Care, v.12, p.113-124, 2002.

NOLAN, J. Fluid resuscitation for the trauma patient. Resuscitation, v.28, p.57-69, 2001

OAKLEY, R.E.; OLIVIER, B.; EYSTER, G.E. et al. Experimental evaluation of central venous pressure monitoring in the dog. J. Am. Anim. Hosp. Assoc., v.33, p.77$82,1997$.

PANKE, W.F.; BRADLEY, E.G.; MORENO, A.H. et al. Technique hazards and usefulness of percutaneous splenic photography. J. Am. Vet. Med. Assoc., v.169, p.1032, 1959.
RABELO, R.C. Avaliações pressóricas arterial, venosas central e periférica, parâmetros clínicos e hematológicos em cães submetidos experimentalmente a eventos hipotensores. 2003. 131f. Tese (Mestrado em Medicina Veterinária) Escola de Veterinária, Universidade Federal de Minas Gerais, Belo Horizonte.

RAISER, A.G. Choque. In: Fundamentos de terapia intensiva veterinária em pequenos animais: conduta do paciente crítico. 1.ed. Rio de Janeiro: L.F. Livros de Veterinária, 2005. p.71-104.

REMILLARD, R.L.; ROSS, J.N.; EDDY, J.B. Variance of indirect blood pressure measurements and prevalence of hypertension in clinically normal dogs. Am. J. Vet. Res., v.52, p.561-565, 1991.

ROODT, A.R.; DOLAB, J.A.; SEGRE, L. Fisiopatologia y diagnostico del ataque por serpientes venenosas: una breve actualización. Rev. Med. Vet., v.77, p.64-71, 1996.

RUDLOFF, E.; KIRBY, R. Fluid therapy. cristalloids and colloids. Vet. Clin. North Am.: Small Anim. Pract., v.28, p.297-329, 1998.

SAMPAIO, I.B.M. Estatística aplicada à experimentação animal. Belo Horizonte: FEPMVZ, 1998. 221p.

SANO-MARTINS, I. S.; SANTORO, M.L.; MORENA, P. et al. Hematological changes induced by Bothrops jararaca venom in dogs. Braz. J. Med. Biol. Res., v.28, p.303-312, 1995.

SILVA JUNIOR, P.G.P. Avaliação clínica e laboratorial de cães tratados com salina tópica, Kalanchoe brasiliensis $e$ soro antibotrópico após o envenenamento botrópico experimental (Bothrops alternatus Duméril, 1854). 2003. 103f. Tese (Mestrado em Medicina Veterinária) - Escola de Veterinária, Universidade Federal de Minas Gerais, Belo Horizonte.

SOARES, P.C.; MELO, G.R.; SANTOS, R.M.B. et al. Pressão venosa central de cães induzidos ao choque hemorrágico, sob efeito de anestesia e repositores hidroeletrolíticos. Rev. Bras. Med. Vet., v.22, p.18-22, 2000.

USER'S guide: statistics. 5.ed. Cary: SAS Institute, 1985. v.1, 956p.

VINCENT, I.C.; MICHELL, A.R.; LEAHY, R.A. Noninvasive measurement of arterial blood pressure in dogs: a potential indicator for the identification of stress. Res. Vet. Sci., v.54, p.195-201, 1993.

WARREN, J.V.; BRANNON, E.S.; STEAD, E.A. et al. The effect of venesection and pooling of blood in the extremities on the atrial pressure and cardiac output in normal subjects with observations on acute circulatory collapse in three instances. J. Clin. Invest., v.24, p.337, 1945.

WIGGERS, C.J. The failure of transfusion in irreversible hemorrhagic shock. Am. J. Physiol., v.116, p.91, 1945.

YEOMANS, A.; PORTER, R.R.; SWANK, R.L. Observations on certain manifestations of circulatory congestion produced in dogs by rapid infusions. J. Clin. Invest., v.22, p.33, 1943. 